\title{
A History-Dependent Cohesive Zone Model in Elastic and Visco-Elastic Materials under Constant and Variable Loading
}

\author{
L. Hakim ${ }^{\mathrm{a}}$, S.E. Mikhailov ${ }^{\mathrm{b}, *}$ \\ ${ }^{a}$ Department of Mathematics, University of Exeter, UK \\ ${ }^{b}$ Department of Mathematics, Brunel University London, UK
}

\begin{abstract}
A history-dependent cohesive zone model approach is used to study the crack behaviour in elastic and visco-elasto materials. The cohesive (yield) stress at the cohesive zone points is related to the nonlinear normalised equivalent stress functional over the stress history at these points, and is expressed in the form of an Abel-type (fractional) integral. We analyse the cohesive zone length evolution in time and the crack tip opening during the stationary crack stage as well as during the propagating crack stage. We consider the external load increasing linearly with time and compare the solution with the case of the constant load. We obtain the solution numerically and analyse the influence of the viscoelasticity by comparing with the case of purely elastic behaviour of the bulk of the material.

Keywords: Cohesive zone, Time-dependent load, Abel-type integral equation, Viscoelasticity
\end{abstract}

\section{Introduction}

An important assumption needed in order to use linear elastic fracture mechanics is that the inelastic region at the crack tip must be negligible in comparison to the size of the whole crack itself. However, in many situations this assumption does not hold. In such cases, elasto-plastic fracture mechanics is considered instead, which particularly includes cohesive zone models. They allow modelling of short crack growth as well as crack nucleation and initiation. In such models, it is assumed that there exists a cohesive zone, CZ, which is the area between two separating but still sufficiently close surfaces ahead of the crack tip, see the shaded region in Fig. 1.

\footnotetext{
${ }^{*}$ Corresponding author

Email address: sergey.mikhailov@brunel.ac.uk (S.E. Mikhailov)
} 


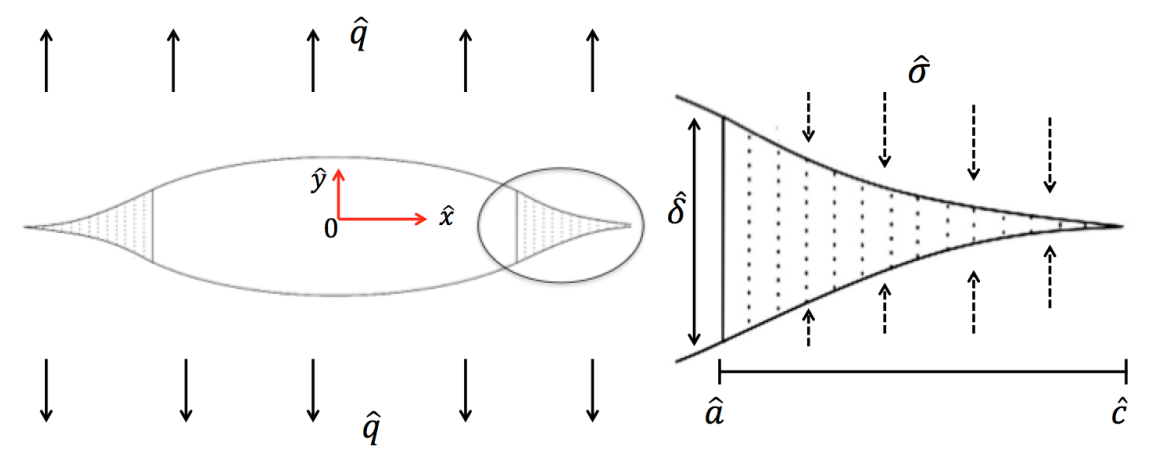

Figure 1: Cohesive zone

At this cohesive zone, cohesive tractions pull the cohesive faces together. The external loads applied to a body cause the crack to propagate and could also cause the crack faces to move further away from each other. By implementing a cohesive zone model, we would like to find out the size of this cohesive zones, and more importantly, when the crack will start to propagate and how fast will it propagate thereafter. When the crack propagates, the cohesive forces vanish at the points where the cohesive zone opening reaches a critical value and these points become the crack surface points, while the new material points, where the history-dependent normalised equivalent stress reaches a critical value, join the cohesive zone. So, the CZ is practically attached to the crack tip ahead of the crack and moves with the crack, keeping the normalised equivalent stress finite in the body.

There have been many cohesive zone models (employing different tractionseparation laws) introduced in the literature. The simplest model, introduced by Leonov-Panasyuk-Dugdale (LPD) (1959-1960), see [3], 8], is when the cohesive stress, that can be associated with the yield stress, $\sigma_{y}$, is constant, while the bulk of the material is elastic. This model is one of the most popular cohesive zone models and many modifications of this model have been made and widely used in nonlinear fracture mechanics.

The 3 main compounds of a cohesive zone model are:

- the constitutive equations in the bulk of the material;

- the constitutive equations in the cohesive zone;

- the criterion for the cohesive zone to break, i.e., the crack to propagate.

The model presented in this paper is an extension of the LPD model to linear visco-elastic behaviour of the bulk of materials with non-linear historydependent constitutive equations in the cohesive zone. Our aim is to find the time evolution of the $\mathrm{CZ}$ before the crack starts propagating, the delay time, after which the crack will start to propagate, and model further the time evolution of the crack and the CZ. In all these stages we assume the quasi-static evolution 
of crack and cohesive zones, i.e., the dynamic effects are not considered. The case of a constant external load was considered in [6], with some preliminary results published in [7]. The focus of this paper is to show that the suggested model and methods for solving the corresponding crack problems are equally applicable to the external external load variable in time.

\section{Problem Formulation}

Let the problem geometry be as in Fig. 1 i.e, the crack occupies the interval $[-\hat{a}(\hat{t}), \hat{a}(\hat{t})]$ and the cohesive zone occupies the intervals $[-\hat{c}(\hat{t}),-\hat{a}(\hat{t})]$ and $[\hat{a}(\hat{t}), \hat{c}(\hat{t})]$ in an infinite linearly elastic or viscoelastic body under plane strain or plain stress conditions. The body is loaded at infinity by traction $\hat{q}(\hat{t})$ in the direction normal to the crack, applied at the time $\hat{t}=0$ and is constant in the coordinate $\hat{x}$. The crack is traction-free, i.e., $\hat{\sigma}_{\hat{y} \hat{y}}(\hat{x}, 0, \hat{t})=0$ for $|\hat{x}|<\hat{a}(\hat{t})$. To simplify the notations, we denote the normal stress ahead of the crack as $\hat{\sigma}$, i.e., $\hat{\sigma}(\hat{x}, \hat{t})=\hat{\sigma}_{\hat{y} \hat{y}}(\hat{x}, 0, \hat{t})$. The initial CZ tip coordinate and crack tip coordinates are prescribed, $\hat{c}(0)=\hat{a}(0)=\hat{a}_{0}$, while the functions $\hat{c}(\hat{t})$ and $\hat{a}(\hat{t})$ for time $\hat{t}>0$ are to be found.

We will now formulate and normalise the principal equations while considering two cases: a constant external load $\hat{q}$ and an external load $\hat{q}(t)$ varying in time.

\subsection{Natural Form of the Cohesive Zone Condition}

First, we will replace the LPD cohesive zone stress condition, $\sigma=\sigma_{y}$, with the history-dependent condition

$$
\underline{\Lambda}(\hat{\boldsymbol{\sigma}} ; \hat{t})=1,
$$

where

$$
\underline{\Lambda}(\hat{\boldsymbol{\sigma}} ; \hat{t})=\left(\frac{\beta}{b \sigma_{0}^{\beta}} \int_{0}^{\hat{t}}|\hat{\boldsymbol{\sigma}}(\hat{\tau})|^{\beta}(\hat{t}-\hat{\tau})^{\frac{\beta}{b}-1} d \hat{\tau}\right)^{\frac{1}{\beta}}
$$

is the normalised history-dependent equivalent stress, $|\hat{\boldsymbol{\sigma}}|$ is the maximum of the principal stresses, and $\hat{t}$ denotes time.

The parameters $\sigma_{0}$ and $b$ are material constants in the assumed power-type relation

$$
\hat{t}_{\infty}(\hat{\sigma})=\left(\frac{\hat{\sigma}}{\sigma_{0}}\right)^{-b}
$$

between the physical rupture time $\hat{t}_{\infty}$ and the constant uniaxial tensile stress $\hat{\sigma}$ applied to a sample without cracks. These parameters can be obtained by fitting the creep durability experimental data on macro-samples. Here, $b$ is dimensionless, $\hat{t}_{\infty}$ has units of time, e.g., seconds, $s$, and if the stress $\hat{\sigma}$ is in Pascals, $\mathrm{Pa}$, then $\hat{\sigma}_{0}$ has units $\mathrm{Pa} \cdot \mathrm{s}^{1 / b}$. For many structural materials the parameter $b$ is in the range between 5 and 20, cf. e.g. [10] and references therein. The dimensionless parameter $\beta$ is a material constant in the nonlinear 
accumulation rule for durability under variable load, see [10]. Further details of this model can be found in [6] and [4].

Note that relations (11)-(2) were implemented in [11] and [5] to solve a similar crack propagation problem without a cohesive zone; i.e. it was assumed that when condition (1) is reached at a point, the crack spreads to this point. However, such approach appeared to be inapplicable for $b \geq 2$. In this paper, a cohesive zone approach is developed instead, in order to cover the larger range of $b$ values relevant to structural materials. In the $\mathrm{CZ}$ approach, when condition (1) is reached at a point, the cohesive zone spreads to this point.

As proved in [6, Section 3.2], the CZ model is applicable only if material parameters, $b$ and $\beta$, of the history dependent yield condition, are such that $b>0,0<\beta<b$. This implies that the CZ model is not applicable for the Robinson-type history-dependent yield condition, based on the power-type durability diagram, for which $\beta=b$.

The cohesive zone condition (1)-(2) at a point $\hat{x}$ on the cohesive zone can be rewritten as

$$
\int_{\hat{t}_{c}(\hat{x})}^{\hat{t}} \hat{\sigma}^{\beta}(\hat{x}, \hat{\tau})(\hat{t}-\hat{\tau})^{\frac{\beta}{b}-1} d \hat{\tau}=\frac{b \sigma_{0}^{\beta}}{\beta}-\int_{0}^{\hat{t}_{c}(\hat{x})} \hat{\sigma}^{\beta}(\hat{x}, \hat{\tau})(\hat{t}-\hat{\tau})^{\frac{\beta}{b}-1} d \hat{\tau},
$$

for $\hat{t} \geq \hat{t}_{c}(\hat{x})$ and $\hat{a}(\hat{t}) \leq|\hat{x}| \leq \hat{c}(\hat{t})$. Here, $\hat{t}_{c}(\hat{x})$ denotes the time when the cohesive zone spreads to the point $\hat{x}$. Equation (4) is an inhomogeneous nonlinear Volterra integral equation of the Abel type (nonlinear fractional integral equation) with unknown function $\hat{\sigma}(\hat{x}, \hat{t})$ for $\hat{t} \geq \hat{t}_{c}(\hat{x})$.

\subsection{Normalised Form of the Cohesive Zone Condition}

To simplify condition (4), and other equations further on, we normalise the variables, which will make them dimensionless and reduce the number of significant parameters. This also make the comparison of the results for constant and variable load cases more illustrative.

For the constant loading case, $\hat{q}(\hat{t})=\hat{q}_{0}$ is independent of time, and using equation (3) we obtain

$$
\hat{t}_{\infty}=\left(\frac{\hat{q_{0}}}{\sigma_{0}}\right)^{-b} .
$$

Then we can introduce the normalised time, coordinate variable, crack tip coordinate, cohesive zone tip coordinate, and stress as follows,

$t=\frac{\hat{t}}{\hat{t}_{\infty}}, \quad x=\frac{\hat{x}}{\hat{a}_{0}}, \quad a(t)=\frac{\hat{a}\left(t \hat{t}_{\infty}\right)}{\hat{a}_{0}}, \quad c(t)=\frac{\hat{c}\left(t \hat{t}_{\infty}\right)}{\hat{a}_{0}}, \quad \sigma(x, t)=\frac{\hat{\sigma}\left(x \hat{a}_{0}, t \hat{t}_{\infty}\right)}{\hat{q}_{0}}$,

and the normalised external load becomes $q(t)=\hat{q}(\hat{t}) / \hat{q}_{0}=1$.

The situation is a bit more complicated when the load is time-dependent, particularly, when it is given by a linear function, i.e., $\hat{q}(\hat{t})=\dot{q} \hat{t}$, with $\dot{q}=$ const. Let us denote by $t_{\bullet} \infty$ the corresponding rupture time, i.e., the time when the cohesive zone spreads over the infinite plane without crack, under the variable 
load. To obtain $\hat{t}_{\bullet}$, we consider equation (4) for $\hat{t}=\hat{t}_{c}$ and $\hat{\sigma}(\hat{x}, \hat{\tau})=\hat{q}(\hat{\tau})=\dot{q} \hat{\tau}$, which reduces to

$$
\dot{q}^{\beta} \int_{0}^{\hat{t}} \hat{\tau}^{\beta}(\hat{t}-\hat{\tau})^{\frac{\beta}{b}-1} d \hat{\tau}=\frac{b \sigma_{0}^{\beta}}{\beta} .
$$

Expressing the integral in $(6)$ in terms of the Beta-function and solving the equation for $\hat{t}=\hat{t}_{\bullet}$ gives

$$
\hat{t}_{\bullet \infty}=\left(\frac{\sigma_{0}}{\dot{q} \alpha}\right)^{\frac{b}{1+b}}
$$

where

$$
\alpha:=\left(\frac{b}{\beta \mathrm{B}\left[\frac{\beta}{b}, 1+\beta\right]}\right)^{-1 / \beta},
$$

and $\mathrm{B}$ is the Beta-function.

For the load linearly increasing in time, the load maximum, reached before rupture in the infinite plane without crack, is evidently $\hat{q}\left(\hat{t}_{\bullet}\right)=\dot{q} \hat{t}_{\bullet}$. Let us also introduce the reference constant load $\hat{q}_{0}=\sigma_{0} \hat{t}_{\bullet \infty}^{-1 / b}$ under which such plane ruptures at the same time $\hat{t}_{\bullet}$. Expressing $\dot{q}$ and $\hat{t}_{\bullet}$ in terms of $\hat{q}\left(\hat{t}_{\bullet}\right)$ and $\hat{q}_{0}$ from these two equations and substituting them in equation (7), we obtain

$$
\hat{q}_{0}=\alpha \hat{q}\left(\hat{t}_{\bullet \infty}\right) .
$$

Now, similarly to the case of constant load, we can introduce the normalised time, coordinate variable, crack tip coordinate, cohesive zone tip coordinate, and stress as follows,

$$
\begin{aligned}
& t=\frac{\hat{t}}{\hat{t}_{\bullet}}, \quad t_{c}=\frac{\hat{t}_{c}}{\hat{t}_{\bullet}}, \quad x=\frac{\hat{x}}{\hat{a}_{0}}, \\
& a(t)=\frac{\hat{a}\left(t \hat{t}_{\bullet \infty}\right)}{\hat{a}_{0}}, \quad c(t)=\frac{\hat{c}\left(t \hat{t}_{\bullet \infty}\right)}{\hat{a}_{0}}, \quad \sigma(x, t)=\frac{\hat{\sigma}\left(x \hat{a}_{0}, t \hat{t}_{\bullet \infty}\right)}{\hat{q}_{0}},
\end{aligned}
$$

and the normalised external load becomes

$$
q(t)=\frac{\hat{q}(\hat{t})}{\hat{q}_{0}}=\frac{\hat{t} \dot{q}}{\hat{q}_{0}}=\frac{t \hat{t}_{\bullet \infty} \dot{q}}{\hat{q}_{0}}=\frac{t}{\alpha}
$$

Hence, after the normalisation, we arrive at the following form of the cohesive zone condition (4) for both the constant and variable load

$$
\int_{t_{c}(x)}^{t} \sigma^{\beta}(x, \tau)(t-\tau)^{\frac{\beta}{b}-1} d \tau=\frac{b}{\beta}-\int_{0}^{t_{c}(x)} \sigma^{\beta}(x, \tau)(t-\tau)^{\frac{\beta}{b}-1} d \tau
$$

for $a(t) \leq|x| \leq c(t)$. 


\subsection{Stress Ahead of the Cohesive Zone}

Let us first consider the case of linear elastic constitutive equations for the bulk of the material. Applying the results by Muskhelishvili (see [12], Section 120), we have for the stresses ahead of the cohesive zone in the elastic material,

$$
\hat{\sigma}(\hat{x}, \hat{t})=\frac{\hat{x}}{\sqrt{\hat{x}^{2}-\hat{c}^{2}(\hat{t})}}\left(\hat{q}(\hat{t})-\frac{2}{\pi} \int_{\hat{a}(\hat{t})}^{\hat{c}(\hat{t})} \frac{\sqrt{\hat{c}^{2}(\hat{t})-\hat{\xi}^{2}}}{\hat{x}^{2}-\hat{\xi}^{2}} \hat{\sigma}(\hat{\xi}, \hat{t}) d \hat{\xi}\right),
$$

for $\hat{t} \leq \hat{t}_{c}(\hat{x})$ and $|\hat{x}|>\hat{c}(\hat{t})$. As one can see from equation (10), $\hat{\sigma}(\hat{x}, \hat{t})$ has generally a square root singularity as $\hat{x}$ tends to $\hat{c}$.

Normalising time, space and stress using equations (5) for the constant load case and (8) for the variable load case, we arrive at the following equation for the normalised stress ahead of the crack tip

$$
\sigma(x, t)=\frac{x}{\sqrt{x^{2}-c^{2}(t)}}\left(q(t)-\frac{2}{\pi} \int_{a(t)}^{c(t)} \frac{\sqrt{c^{2}(t)-\xi^{2}}}{x^{2}-\xi^{2}} \sigma(\xi, t) d \xi\right) \text { for }|x|>c(t)
$$

where

$$
q(t)= \begin{cases}1 & \text { for a constant load } \\ \frac{t}{\alpha} & \text { for a variable load }\end{cases}
$$

\subsection{The Stress Intensity Factor}

A sufficient condition for the normalised equivalent stress, $\Lambda$, to be bounded at the cohesive zone tip is that the stress $\hat{\sigma}$ is bounded, while the necessary condition for the latter is that the stress intensity factor, $\hat{K}$, is zero there. Multiplying the stress in equation 10 by $\sqrt{\hat{x}-\hat{c}(\hat{t})}$ and taking the limit as $\hat{x}$ tends to $\hat{c}(\hat{t})$ yields

$$
\hat{K}(\hat{c}(\hat{t}), \hat{t})=\sqrt{\frac{\hat{c}(\hat{t})}{2}}\left(\hat{q}(\hat{t})-\frac{2}{\pi} \int_{\hat{a}(\hat{t})}^{\hat{c}(\hat{t})} \frac{\hat{\sigma}(\hat{\xi}, \hat{t})}{\sqrt{\hat{c}^{2}(\hat{t})-\hat{\xi}^{2}}} d \hat{\xi}\right) .
$$

In the constant loading case, we will use normalisation relations (5) as well as the following normalisation for the stress intensity factor

$$
K(c, t)=\frac{\hat{K}\left(c \hat{a}_{0}, t \hat{t}_{\infty}\right)}{\hat{q}_{0} \sqrt{\hat{a}_{0}}}
$$

while, in the variable loading case, we will use normalisation relations 8 as well as the following normalisation for the stress intensity factor

$$
K(c, t)=\frac{\hat{K}\left(c \hat{a}_{0}, t \hat{t}_{\bullet}\right)}{\hat{q}_{0}(\hat{t}) \sqrt{\hat{a}_{0}}}
$$


Finally, this leads to the following normalised equation for the stress intensity factor

$$
K(c(t), t)=\sqrt{\frac{c(t)}{2}}\left(q(t)-\frac{2}{\pi} \int_{a(t)}^{c(t)} \frac{\sigma(\xi, t)}{\sqrt{c^{2}(t)-\xi^{2}}} d \xi\right) .
$$

where $q(t)$ is given by 12 .

\subsection{Objectives}

Further on we will study the evolution of the cohesive zone length with time during two stages: (i) stationary crack stage; and (ii) propagating crack stage. In each of these two stages, we will consider two models for the bulk of the material: (a) linear elasticity and (b) linear viscoelasticity. The main parameters involved in the CZM equations are $b$ and $\beta$.

We will compare the results for the external load varying in time and for the constant one. The suitability of the numerical method used in these problems has been analysed in [6] and [4, where mesh-refinements and the numerical convergence rates were considered.

\section{Cohesive Zone Growth for a Stationary Crack}

In this section we will consider the stationary crack stage, before the crack starts propagating, i.e., $a(t)=a(0)=1$, and thus only the cohesive zone grows with time.

The details of the algorithm used to find the cohesive zone length with respect to time can be found in Section 4.1 in [6. Briefly, we introduce a time mesh with time steps $t_{i}$. At each time step $t_{i}$, we solve equation $K\left(c_{i}, t_{i}\right)=0$ to obtain $c\left(t_{i}\right)$.

In order to evaluate the integral in $[13$, we need to find the stress in the cohesive zone as well as the cohesive zone tip coordinate. To that end, we solve integral equation 9 for $\sigma^{\beta}\left(x, t_{i}\right)$ when $x=c\left(t_{k}\right), k=0,1,2,3, \ldots, i-1$, where $t_{c}(x)=t_{k}$, employing analytical solution of the generalised Abel-type integral equation. As the right hand side of equation (9) depends on the stresses ahead of the cohesive zone tip, we use equation (11) to obtain $\sigma^{\beta}(x, t)$ for $x>c(t)$, when $t \leq t_{k}$.

All programming in this section and further on was implemented in MATLAB. The integrals were evaluated in terms of the Gauss hypergeometric function ${ }_{2} F_{1}$. We were solving the problem for several different $b$ and for $\beta=b / 2$, $\beta=b / 3, \beta=b / 4, \beta=b / 6$ or $\beta=b / 8$ (for such choices of $\beta$ the Gauss hypergeometric functions can be represented in a simple analytical form). Note that this limitation on $\beta$ choices is not essential and can be avoided if an appropriate general numerical integration scheme is used for approximating the weakly-singular Abel-type integrals.

Using the outlined numerical scheme, we obtained the evolution of the cohesive zone tip position as well as the stress distribution on the cohesive zone. 


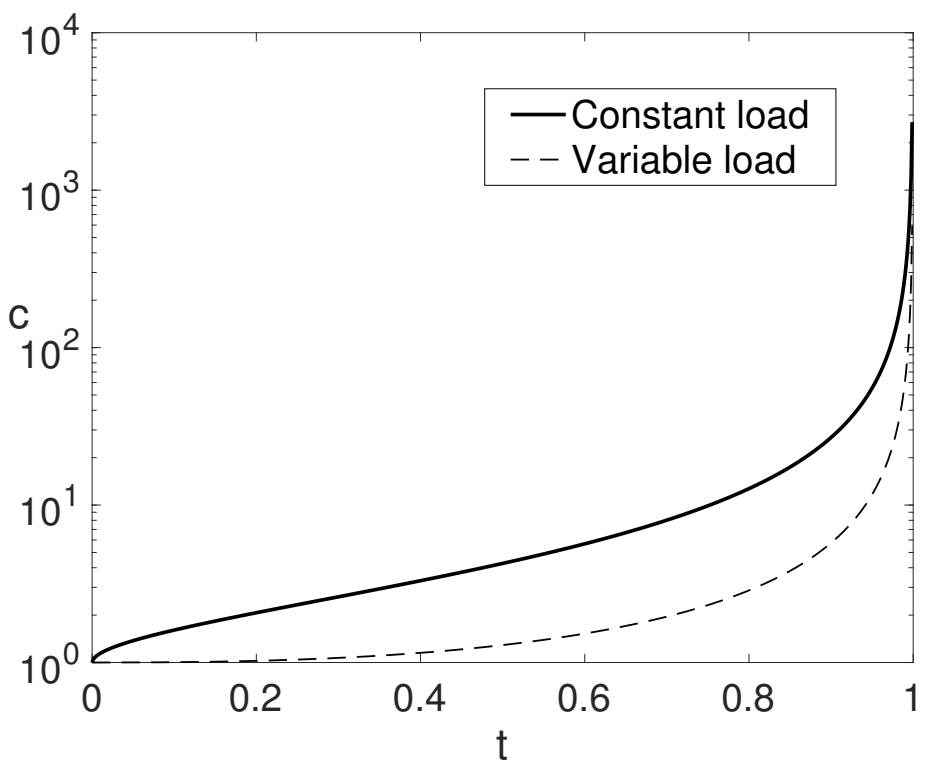

Figure 2: $\mathrm{CZ}$ tip coordinate vs. time for $b=4, \beta=2$

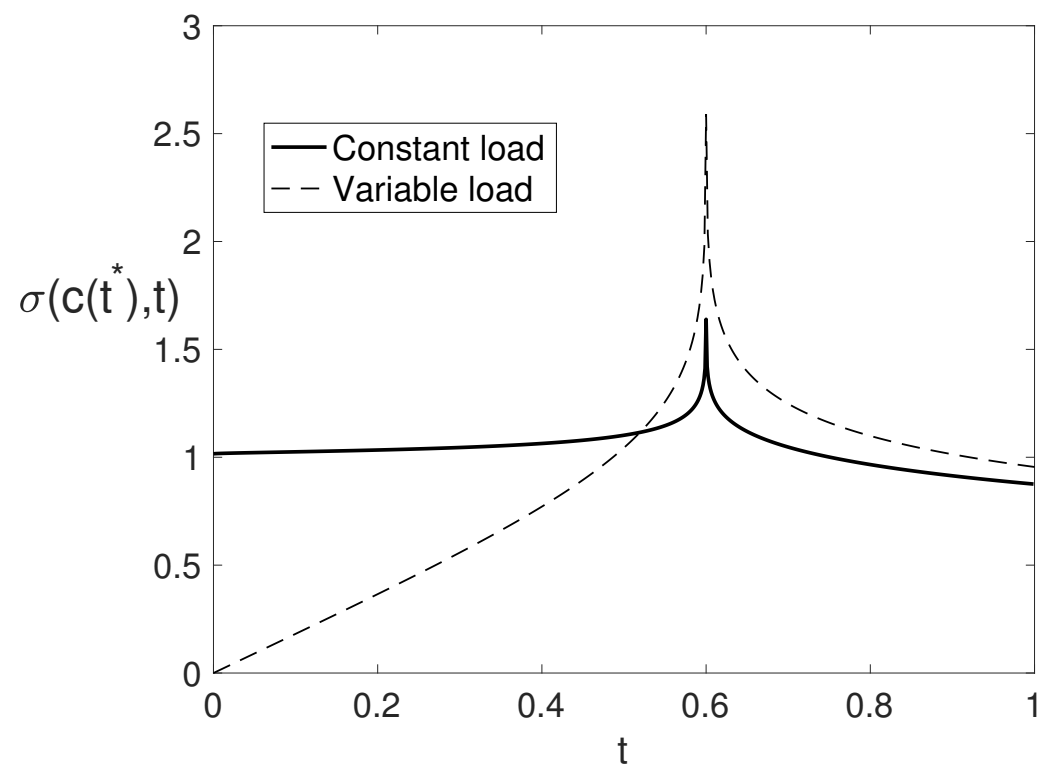

Figure 3: $\sigma\left(c\left(t^{*}\right), t\right)$ for $b=4, \beta=2$ at $t^{*}=0.6$ 
The curves in Fig. 2 show that the cohesive zone length ahead of the stationary crack is monotonically and continuously increasing with time. We can also conclude that the $\mathrm{CZ}$ tip coordinate grows more rapidly with time in the constant loading case than in the variable loading case. From Fig. 3 we can see that the stress at a material point ahead of the cohesive zone is growing monotonically in time $t$, peaking at the time $t^{*}$ when the point becomes the $\mathrm{CZ}$ tip, and then monotonically decreasing inside the CZ.

Note that on the stationary crack stage, the cohesive zone evolution and stress distribution are the same for the elastic and visco-elastic models and do not depend on the elastic constants or visco-elastic operators in the considered problem.

\section{Crack Tip Opening}

We will first consider the case when the bulk of the material is linearly elastic and then convert the obtained solution to the case of linear visco-elastic materials using the so-called Volterra principle.

\subsection{Linear Elastic Material}

The crack opening is calculated similar to the classical LPD model in [8, 3] and is presented as

$$
\left.\left[\hat{u}_{e}\right](\hat{x}, \hat{t})=\left[\hat{u}_{e}^{(\hat{q})}\right](\hat{x}, \hat{t})+\left[\hat{u}_{e}^{(\hat{\sigma})}\right](\hat{x}, \hat{t})\right],
$$

where

$$
\left[\hat{u}_{e}^{(\hat{q})}\right](\hat{x}, \hat{t})=-\frac{\varkappa+1}{2 \pi \mu} \hat{q}(\hat{t}) \int_{-\hat{c}(\hat{t})}^{\hat{c}(\hat{t})} \widetilde{\Gamma}(\hat{x}, \hat{\xi} ; \hat{c}(\hat{t})) d \hat{\xi}
$$

and

$\left[\hat{u}_{e}^{(\hat{\sigma})}\right](\hat{x}, \hat{t})=\frac{\varkappa+1}{2 \pi \mu}\left(\int_{-\hat{c}(\hat{t})}^{-\hat{a}(\hat{t})} \hat{\sigma}(\hat{\xi}, \hat{t}) \widetilde{\Gamma}(\hat{x}, \hat{\xi} ; \hat{c}(\hat{t})) d \hat{\xi}+\int_{\hat{a}(\hat{t})}^{\hat{c}(\hat{t})} \hat{\sigma}(\hat{\xi}, \hat{t}) \widetilde{\Gamma}(\hat{x}, \hat{\xi} ; \hat{c}(\hat{t})) d \hat{\xi}\right)$.

In the above expressions, $\mu=E /[2(1+\nu)]$ is the shear modulus, $E$ is Young's modulus of elasticity and $\nu$ is Poisson's ratio, $\varkappa=3-4 \nu$ under the plain strain conditions, while $\varkappa=(3-\nu) /(1+\nu)$ under the plain stress conditions, and finally,

$$
\widetilde{\Gamma}(\hat{x}, \hat{\xi} ; \hat{c}(\hat{t}))=\ln \left[\frac{\hat{c}^{2}(\hat{t})-\hat{x} \hat{\xi}-\sqrt{\left(\hat{c}^{2}(\hat{t})-\hat{x}^{2}\right)\left(\hat{c}^{2}(\hat{t})-\hat{\xi}^{2}\right)}}{\hat{c}^{2}(\hat{t})-\hat{x} \hat{\xi}+\sqrt{\left(\hat{c}^{2}(\hat{t})-\hat{x}^{2}\right)\left(\hat{c}^{2}(\hat{t})-\hat{\xi}^{2}\right)}}\right] .
$$

After integrating 14 and combining the integrals ranging from $-\hat{c}(\hat{t})$ to $-\hat{a}(\hat{t})$ and $\hat{a}(\hat{t})$ to $\hat{c}(\hat{t})$ in $(15)$, we obtain

$$
\left[\hat{u}_{e}^{(\hat{q})}\right](\hat{x}, \hat{t})=\frac{\hat{q}(\hat{t})(1+\varkappa)}{2 \mu} \sqrt{\hat{c}^{2}(\hat{t})-\hat{x}^{2}},
$$


where

$$
\left[\hat{u}_{e}^{(\hat{\sigma})}\right](\hat{x}, \hat{t})=\frac{\varkappa+1}{2 \pi \mu}\left(\int_{\hat{a}(\hat{t})}^{\hat{c}(\hat{t})} \hat{\sigma}(\hat{\xi}, \hat{t}) \Gamma(\hat{x}, \hat{\xi} ; \hat{c}(\hat{t})) d \hat{\xi}\right)
$$

$$
\Gamma(\hat{x}, \hat{\xi} ; \hat{c}(\hat{t}))=\ln \left[\frac{2 \hat{c}^{2}(\hat{t})-\hat{\xi}^{2}-\hat{x}^{2}-2 \sqrt{\left(\hat{c}^{2}(\hat{t})-\hat{x}^{2}\right)\left(\hat{c}^{2}(\hat{t})-\hat{\xi}^{2}\right)}}{2 \hat{c}^{2}(\hat{t})-\hat{\xi}^{2}-\hat{x}^{2}+2 \sqrt{\left(\hat{c}^{2}(\hat{t})-\hat{x}^{2}\right)\left(\hat{c}^{2}(\hat{t})-\hat{\xi}^{2}\right)}}\right] .
$$

We can remark that $\Gamma(\hat{x}, \hat{\xi} ; \hat{c}(\hat{t}))<0$ when $\hat{x}^{2}, \hat{\xi}^{2}<\hat{c}^{2}(\hat{t})$.

The crack tip opening occurs at $x=a(t)$ and therefore is

$\hat{\delta}_{e}(\hat{t}):=\left[\hat{u}_{e}\right](\hat{a}(\hat{t}), \hat{t})=\frac{1+\varkappa}{2 \mu}\left(\hat{q} \sqrt{\hat{c}^{2}(\hat{t})-\hat{a}^{2}(\hat{t})}+\frac{1}{\pi} \int_{\hat{a}(\hat{t})}^{\hat{c}(\hat{t})} \hat{\sigma}(\hat{\xi}, \hat{t}) \Gamma(\hat{a}(\hat{t}), \hat{\xi} ; \hat{c}(\hat{t})) d \hat{\xi}\right)$.

Using the space, time, and stress normalisation given previously, we will apply the normalisations

$$
\delta_{e}(t)=\frac{8 \mu \hat{\delta}_{e}\left(t \hat{t}_{\infty}\right)}{\hat{q}_{0} \hat{a}_{0}(1+\varkappa)} \quad \text { and } \quad \delta_{e}(t)=\frac{8 \mu \hat{\delta}_{e}\left(t \hat{t}_{\bullet}\right)}{\hat{q}_{0} \hat{a}_{0}(1+\varkappa)},
$$

for the constant and varying load cases, respectively. Consequently, we have the following formula for the normalised crack tip opening

$$
\delta_{e}(t)=\frac{4}{\pi}\left(q(t) \pi \sqrt{c(t)^{2}-a(t)^{2}}+\int_{a(t)}^{c(t)} \sigma(\xi, t) \Gamma(a(t), \xi ; c(t)) d \xi\right) .
$$

where, as before, $q(t)$ is given by 12 .

\subsection{Linear Viscoelastic Material}

To obtain the crack tip opening in the viscoelastic case, we will implement the so-called Volterra principle, according to which we have to replace the elastic constants $\mu$ and $\nu$ in the elastic solution by the corresponding viscoelastic operators, to arrive at the viscoelastic solution. Although this approach is not always applicable to viscoelastic problems with moving boundaries, it is possible to show, see [14, that this approach leads to a viscoelastic solution for the plane symmetric problem with a straight propagating crack. This particularly means that the stress representation ahead of the CZ tip following from the results by Muskhelishvili, see [12], and given by equation [11), which does not include the elastic constants at all, is valid also for the considered viscoelastic problem.

For simplicity, we will consider the viscoelastic material with constant (purely elastic) Poisson's ratio $\nu$ (and thus the parameter $\varkappa$ will also remain constant). Then, to obtain the crack opening in the viscoelastic case, we have to replace $\frac{1}{\mu}$ in 17 by the second kind Volterra integral operator $\boldsymbol{\mu}^{-1}$ defined by

$$
\left(\boldsymbol{\mu}^{-1} \hat{\sigma}\right)(\hat{t})=\frac{\hat{\sigma}(\hat{t})}{\mu}+\int_{0}^{\hat{t}} \dot{J}(\hat{t}-\hat{\tau}) \hat{\sigma}(\hat{\tau}) d \hat{\tau}
$$


$\mu$ is the instant shear modulus $J$ is the creep function. To this end, the crack opening in the viscoelastic case can be presented as

$$
\hat{\delta}_{v}(\hat{t})=\left(\boldsymbol{\mu}^{-1} \mu \hat{\delta}_{e}\right)(\hat{t})=\hat{\delta}_{e}(\hat{t})+\mu \int_{0}^{\hat{t}} \dot{J}(\hat{t}-\hat{\tau}) \hat{\delta}_{e}(\hat{\tau}) d \hat{\tau} .
$$

We will further use the creep function of a standard linear solid,

$$
J(\hat{t}-\hat{\tau})=\frac{1}{\mu}+\frac{\hat{\theta}}{\eta}\left(1-e^{-\frac{1}{\hat{\theta}}(\hat{t}-\hat{\tau})}\right),
$$

and so

$$
\dot{J}(\hat{t}-\hat{\tau})=\frac{1}{\eta} e^{-\frac{1}{\theta}(\hat{t}-\hat{\tau})},
$$

where $\hat{\theta}$ denotes the relaxation time and $\eta$ the viscosity. So, equation 20 becomes

$$
\hat{\delta}_{v}(\hat{t})=\hat{\delta}_{e}(\hat{t})+\frac{\mu}{\eta} \int_{0}^{\hat{t}} e^{-\frac{1}{\theta}(\hat{t}-\hat{\tau})} \hat{\delta}_{e}(\hat{\tau}) d \hat{\tau} .
$$

We further use the same normalisations as before to normalise space, time, stress. Furthermore, for the elastic crack tip opening we use normalisation (18), the similar formulas to normalise the viscoelastic crack tip opening as follows,

$$
\delta_{v}(t)=\frac{8 \mu \hat{\delta}_{v}\left(t \hat{t}_{\infty}\right)}{\hat{q}_{0} \hat{a}_{0}(1+\varkappa)} \quad \text { and } \quad \delta_{v}(t)=\frac{8 \mu \hat{\delta}_{v}\left(t \hat{t}_{\bullet \infty}\right)}{\hat{q}_{0} \hat{a}_{0}(1+\varkappa)}
$$

for the constant and varying load cases, respectively. Consequently, the normalised crack tip opening for the viscoelastic case is given by

$$
\delta_{v}(t)=\delta_{e}(t)+m \int_{0}^{t} e^{-\frac{(t-\tau)}{\theta}} \delta_{e}(\tau) d \tau
$$

where $m=\frac{\mu \hat{t}_{\infty}}{\eta}, \theta=\frac{\hat{\theta}}{\hat{t}_{\infty}}$ for the constant load and $m=\frac{\mu \hat{t}_{\bullet}}{\eta}, \theta=\frac{\hat{\theta}}{\hat{t}_{\bullet}}$ for the variable load, and these parameters are dimensionless.

Implementing the integration procedure described in [6], we obtained numerical results for the crack tip opening in a viscoelastic material with parameters

$$
\theta=1, m=5,
$$

that can be attributed to PMMA (cf. Appendix A in [6]), and compared them with the elastic material case, see Fig. 4 


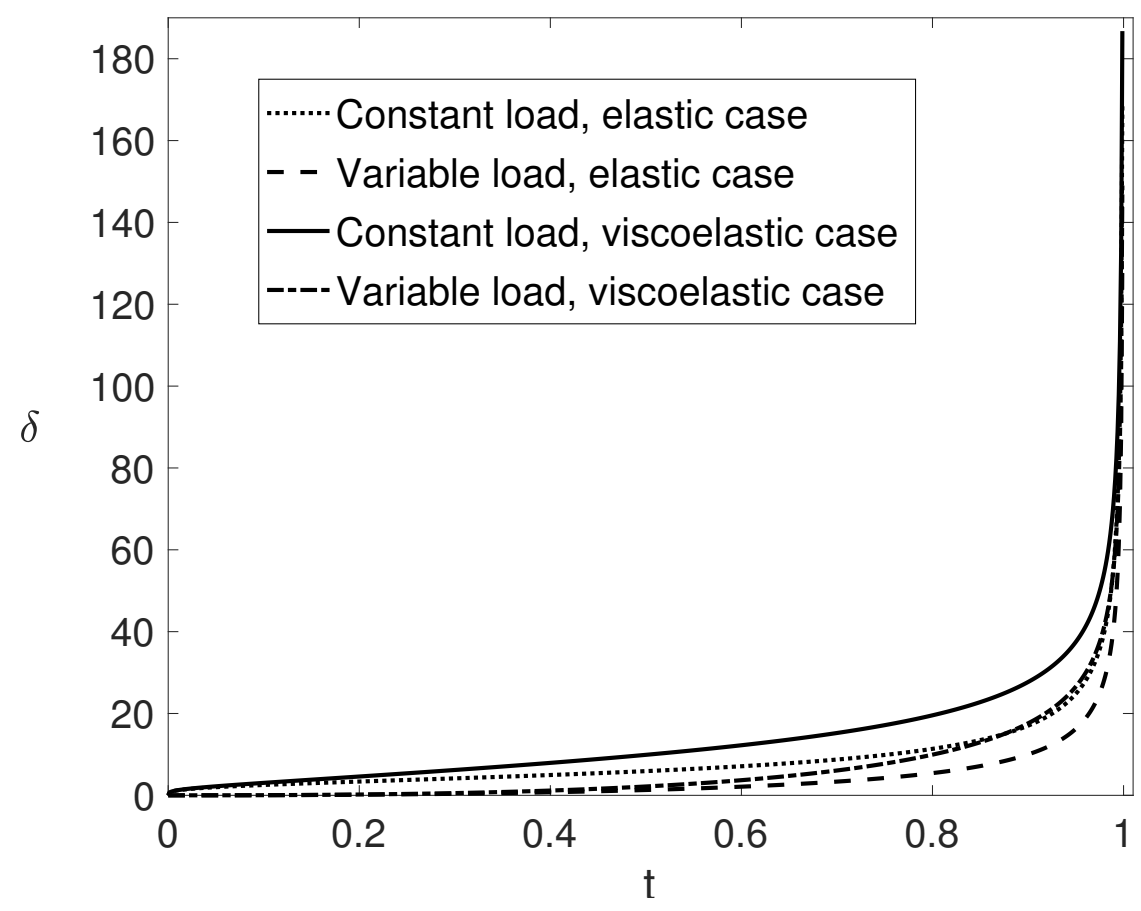

Figure 4: Crack opening vs. time for viscoelastic and elastic materials with $b=4$, $\beta=2, m=5, \theta=1$.

\section{Cohesive Zone Growth for a Propagating Crack}

\subsection{Crack Growth Criterion}

As in the LPD model, we assume that the crack starts to propagate when the crack tip opening $\hat{\delta}$ reaches a critical value $\hat{\delta}_{c}$. For example, the experimentally determined value for PMMA is $\hat{\delta}_{c}=0.0016 \mathrm{~mm}$, see [2, Section 10.3.2]. Using the normalisations given in equation (18) and parameters

$$
\mu / \hat{q}_{0}=23, \varkappa=1.6, \hat{a}_{0}=0.1 \mathrm{~mm},
$$

the normalised critical crack tip opening becomes

$$
\delta_{c} \approx 1.13 .
$$

The time instant, when the crack tip opening reaches a critical value, will be referred to as the fracture delay time and denoted by $\hat{t}_{d}$, where $t_{d}$ corresponds to the normalised delay time.

In terms of the normalised crack tip openings, the crack begins to grow when $\delta(t)$ reaches the critical value $\delta_{c}$. During the crack propagation stage, the crack tip opening satisfies equation

$$
\delta_{e}(t)=\delta_{c}, \quad t \geq t_{d}
$$


for the purely elastic case; and

$$
\delta_{v}(t)=\delta_{c}, \quad t \geq t_{d}
$$

for the viscoelastic case. In the crack propagation stage, the crack length $a(t)$ varies with time and is no longer a constant value. The next section will explain the algorithm used to study how the crack length and CZ length evolve with time.

\subsection{Numerical Algorithm}

The crack length $a$ is equal to 1 for all steps $t<t_{d}$, and at $t=t_{d}$ the crack length begins to grow. The first aim in the crack propagation stage is to find the delay time, $t_{d}$. This is done by solving equations (27) or (28) using the secant method, while $a\left(t_{d}\right)=1$; the corresponding value of $c\left(t_{d}\right)$ is obtained by setting the stress intensity factor at the CZ tip to zero.

To calculate the crack length and the $\mathrm{CZ}$ length at $t>t_{d}$, we use a uniform time mesh with time steps $t_{i}=t_{d}+i \cdot h$, where $h$ is the same step size used during the stationary crack stage. We then implement an iterative method to solve equation (27) (in the elastic case) or 28) (in the visco-elastic case) for $a\left(t_{i}\right)$. To this end, we need $c\left(t_{i}\right)$ at each iteration, and this is obtained by setting the stress intensity factor at the $\mathrm{CZ}$ to zero. Thus we have the following equations to be solved. $\mathrm{q}(\mathrm{t})$

(a) The condition $\underline{\Lambda}(\sigma(x), t)=1$, i.e.,

$$
\int_{t_{c}(x)}^{t} \sigma^{\beta}(x, \tau)(t-\tau)^{\frac{\beta}{b}-1} d \tau=\frac{b}{\beta}-\int_{0}^{t_{c}(x)} \sigma^{\beta}(x, \tau)(t-\tau)^{\frac{\beta}{b}-1} d \tau,
$$

for $a(t) \leq|x| \leq c(t), t>t_{c}(x)$.

(b) The stress ahead of the CZ tip,

$$
\sigma(x, t)=\frac{x}{\sqrt{x^{2}-c^{2}(t)}}\left(q(t)-\frac{2}{\pi} \int_{a(t)}^{c(t)} \frac{\sqrt{c^{2}(t)-\xi^{2}}}{x^{2}-\xi^{2}} \sigma(\xi, t) d \xi\right),
$$

for $|x|>c(t)$.

(c) The zero stress intensity factor at the CZ tip,

$$
K(t, a(t), c(t))=\sqrt{\frac{c(t)}{2}}\left(q(t)-\frac{2}{\pi} \int_{a(t)}^{c(t)} \frac{\sigma(\xi, t)}{\sqrt{c^{2}(t)-\xi^{2}}} d \xi\right) .
$$

(d) Setting the crack tip opening to the critical value

$$
\begin{aligned}
& U(t, a(t), c(t))=\delta_{e}(t)-\delta_{c}=0 \quad \text { for the elastic case } \\
& U(t, a(t), c(t))=\delta_{v}(t)-\delta_{c}=0 \quad \text { for the viscoelastic case. }
\end{aligned}
$$

The details of the algorithm used for finding the length of the crack with respect to time, and the corresponding $\mathrm{CZ}$ length at that time, can be found in Section 6.1 in 6 . 


\subsection{Numerical results}

We will look at the elastic as well as the viscoelastic bulk of the material under constant and variable external loads, with some material parameters given in (24)-(26), while the values of $b$ and $\beta$ are presented in figure legends and captions.

Graphs for the dependence of the delay time and corresponding rupture time on the material model parameters $b$ and $\beta$ are given in Figs. 5 and 6 , for the case of variable loading. Figs. 5 shows the results for fixed $b=4$ and $\beta=b / 8$, $\beta=b / 6, \beta=b / 4, \beta=b / 3, \beta=b / 2$. Fig. 6 illustrates the results for fixed $\beta=1 / 2$ and for $b=8 \beta, b=6 \beta, b=4 \beta, b=3 \beta, b=2 \beta$.

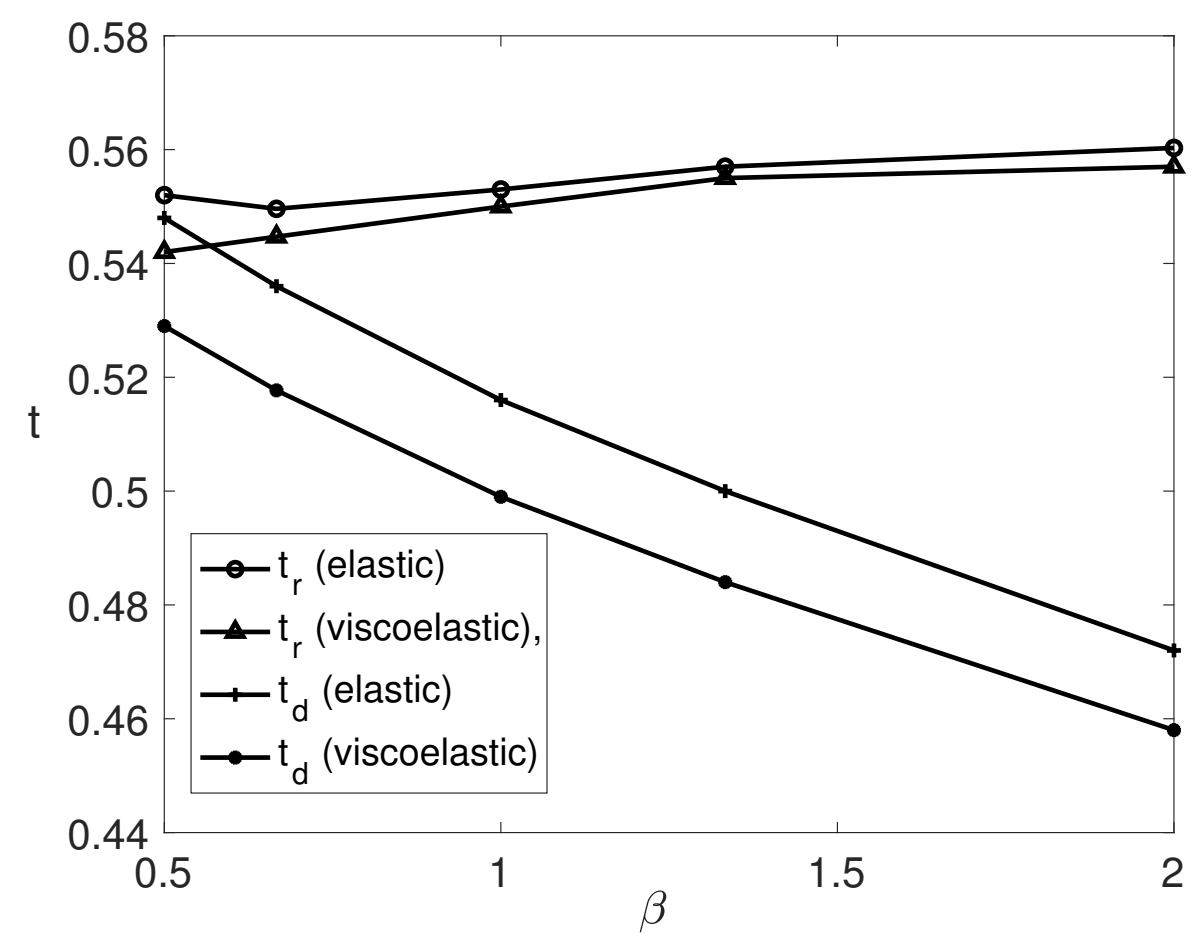

Figure 5: Delay time and rupture time vs. $\beta$ for $b=4$ (variable load) 


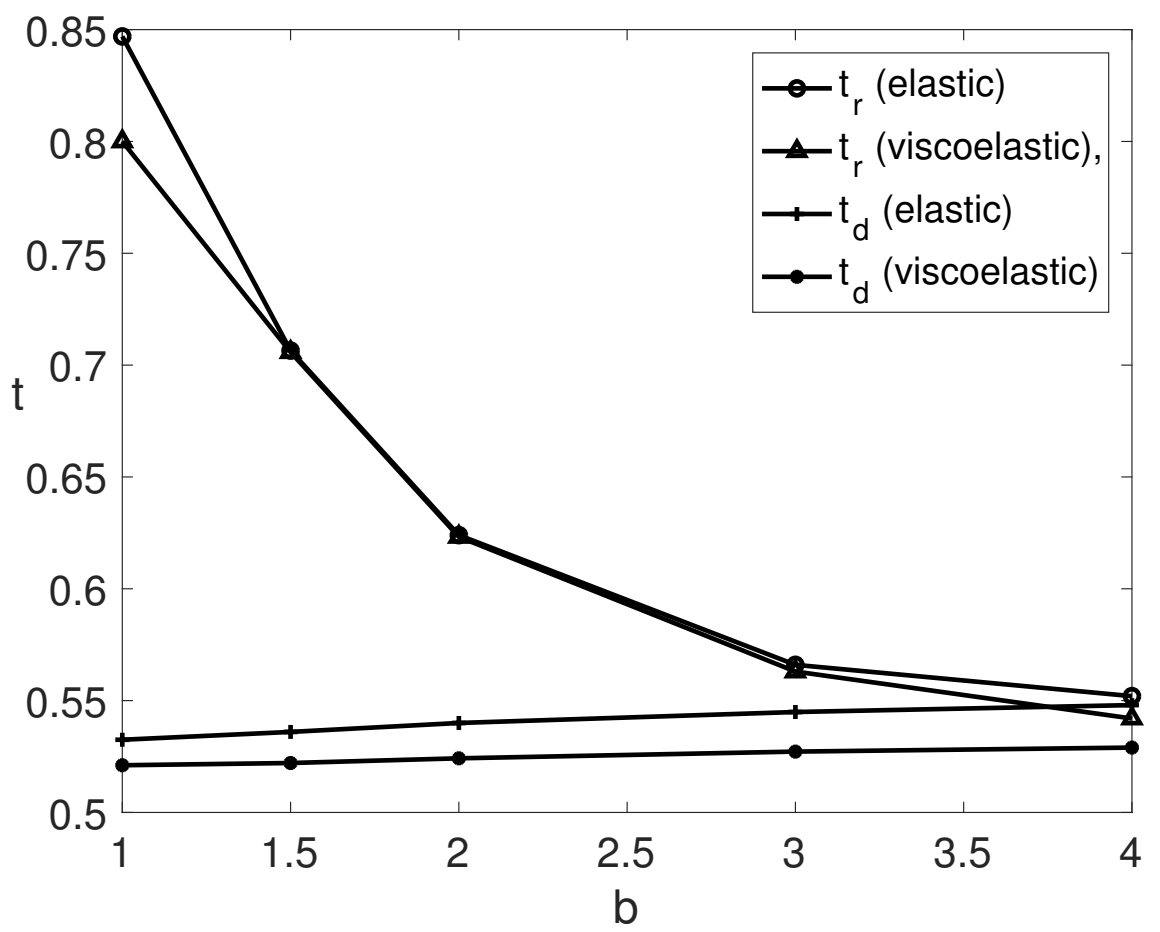

Figure 6: Delay time and rupture time vs. $b$ for $\beta=1 / 2$ (variable load).

Figs. 7] 10 show the evolution of the crack length in time. 


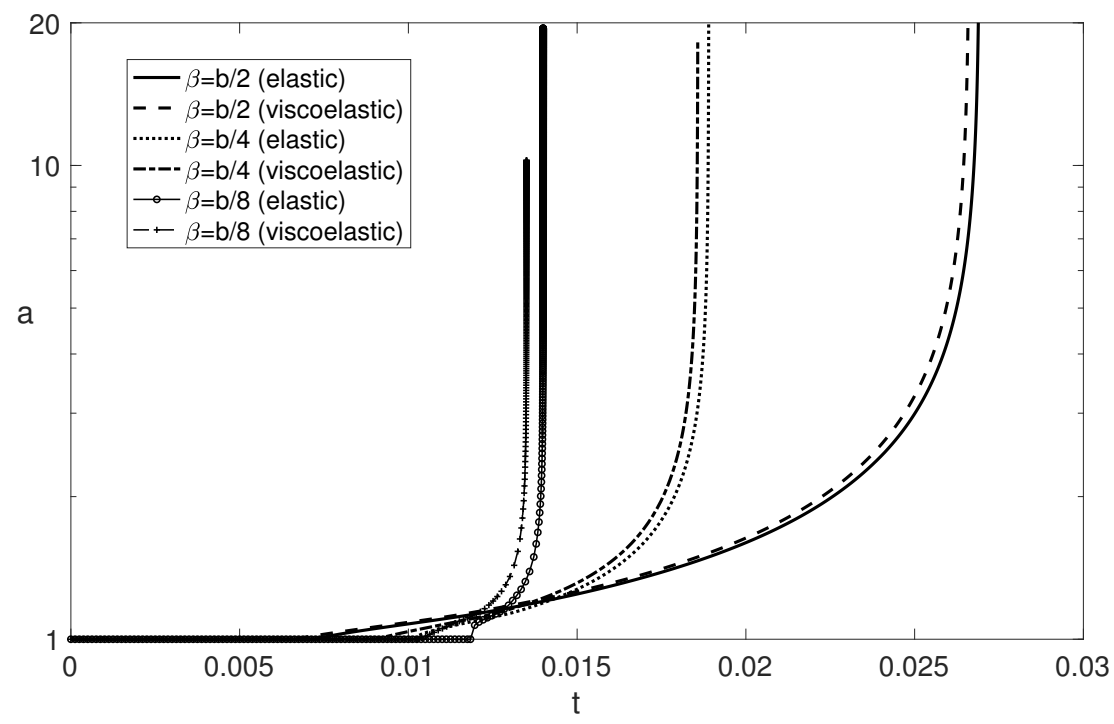

Figure 7: Crack length vs. time for $b=4$, constant load.

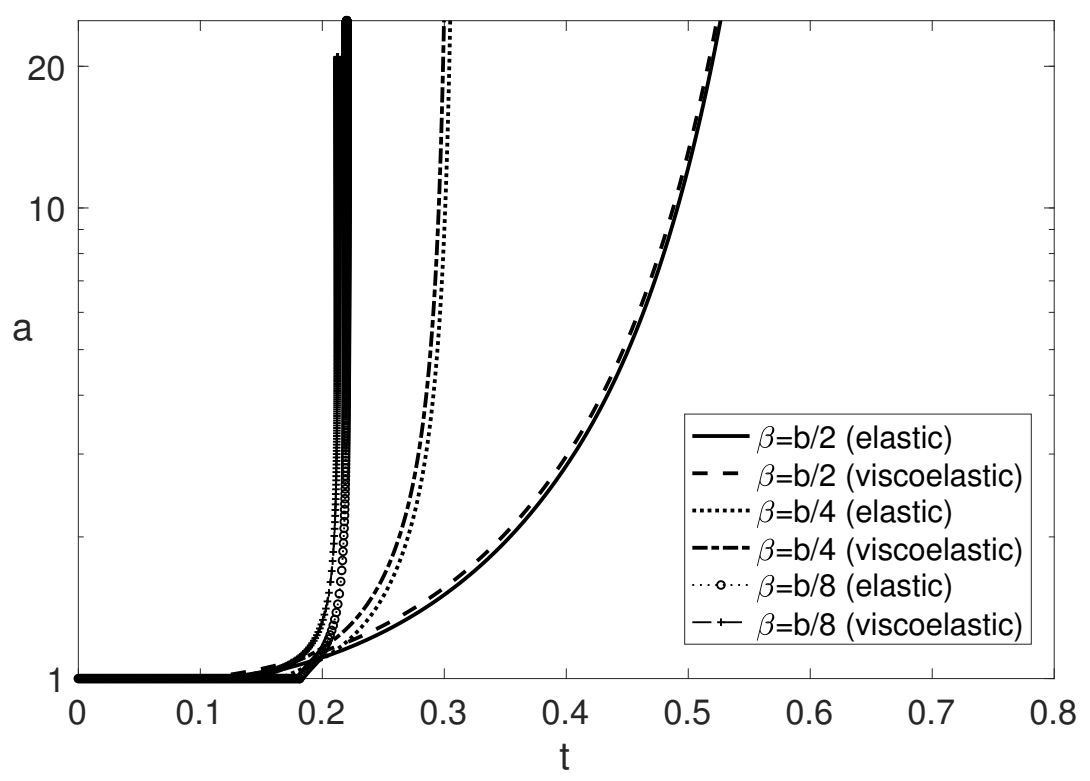

Figure 8: Crack length vs. time for $b=1.5$, constant load. 


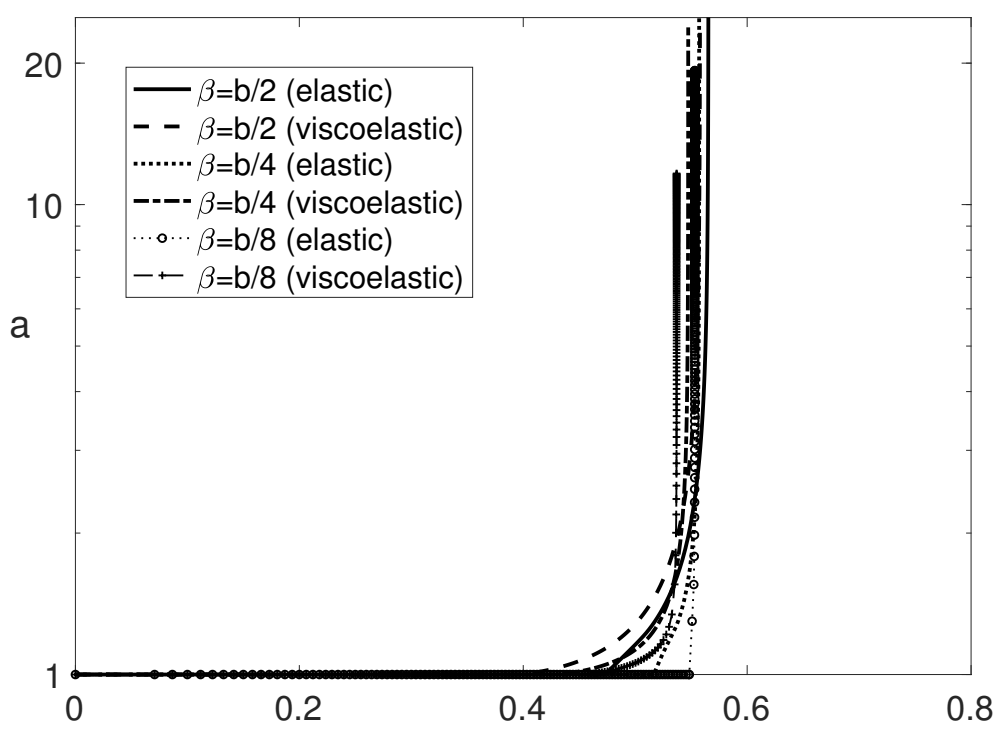

Figure 9: Crack length vs. time for $b=4$, variable load.

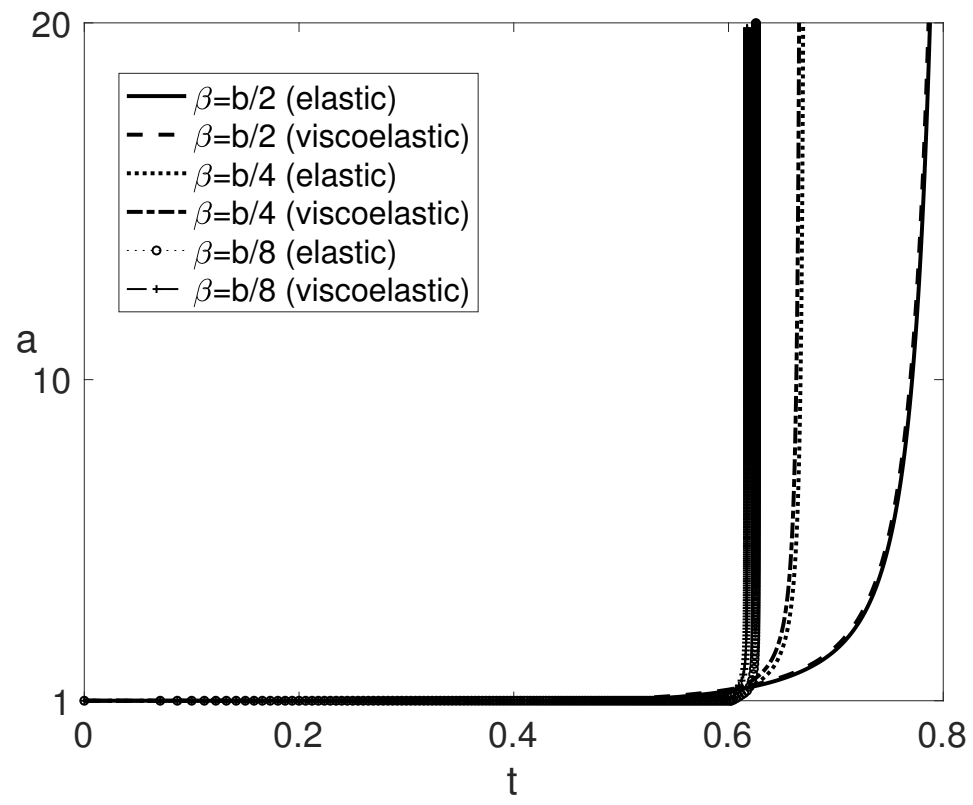

Figure 10: Crack length vs. time for $b=1.5$, variable load. 
Figs. 11 14 show the evolution of the cohesive zone length, $l(t)=c(t)-a(t)$, in time. If the crack propagates faster than the cohesive zone, then eventually the cohesive zone will vanish.

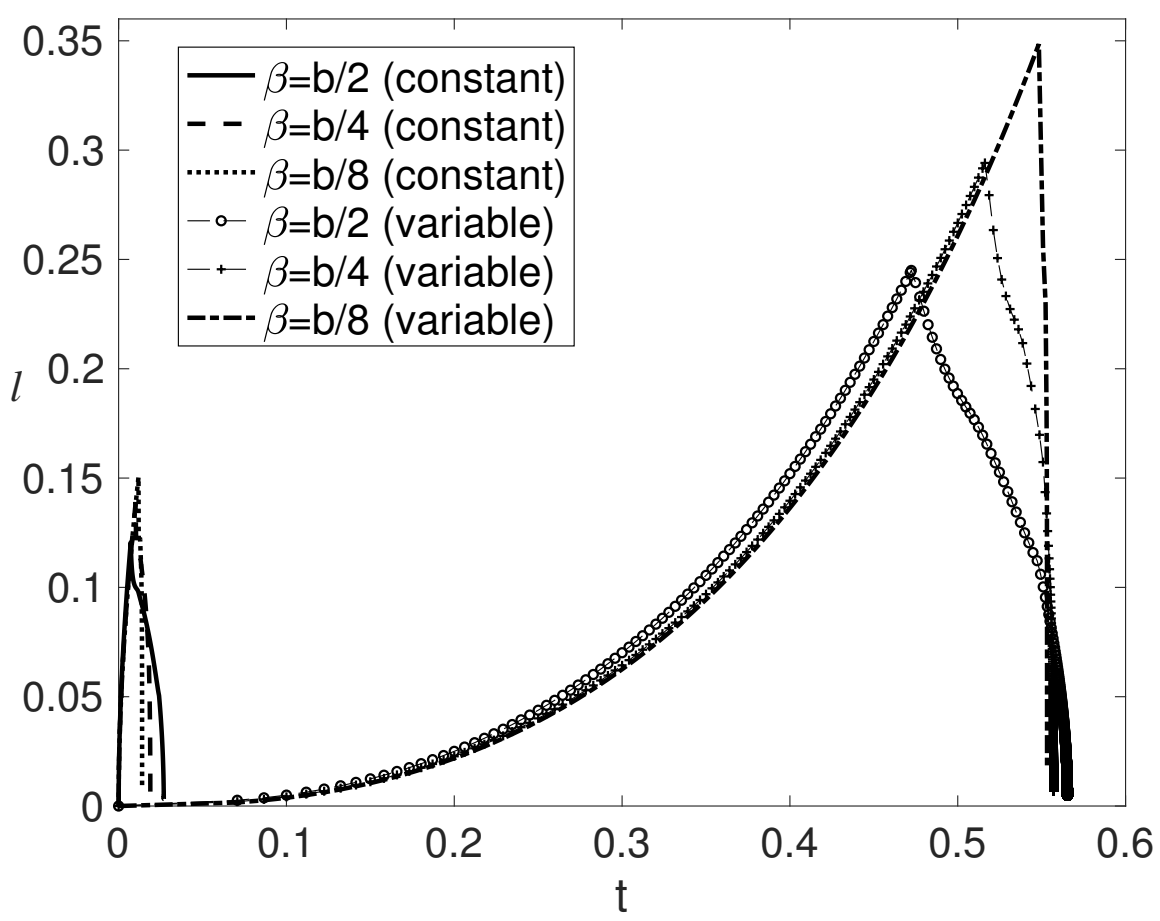

Figure 11: CZ length vs. time for $b=4$ (elastic case). 


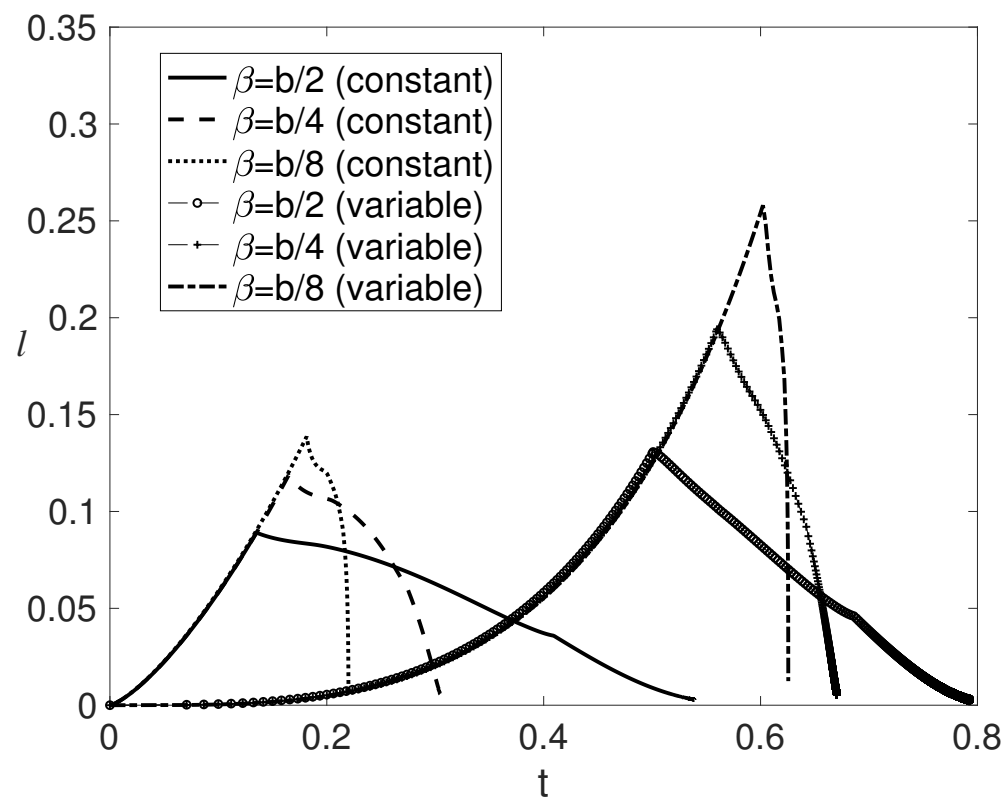

Figure 12: CZ length vs. time for $b=1.5$ (elastic case).

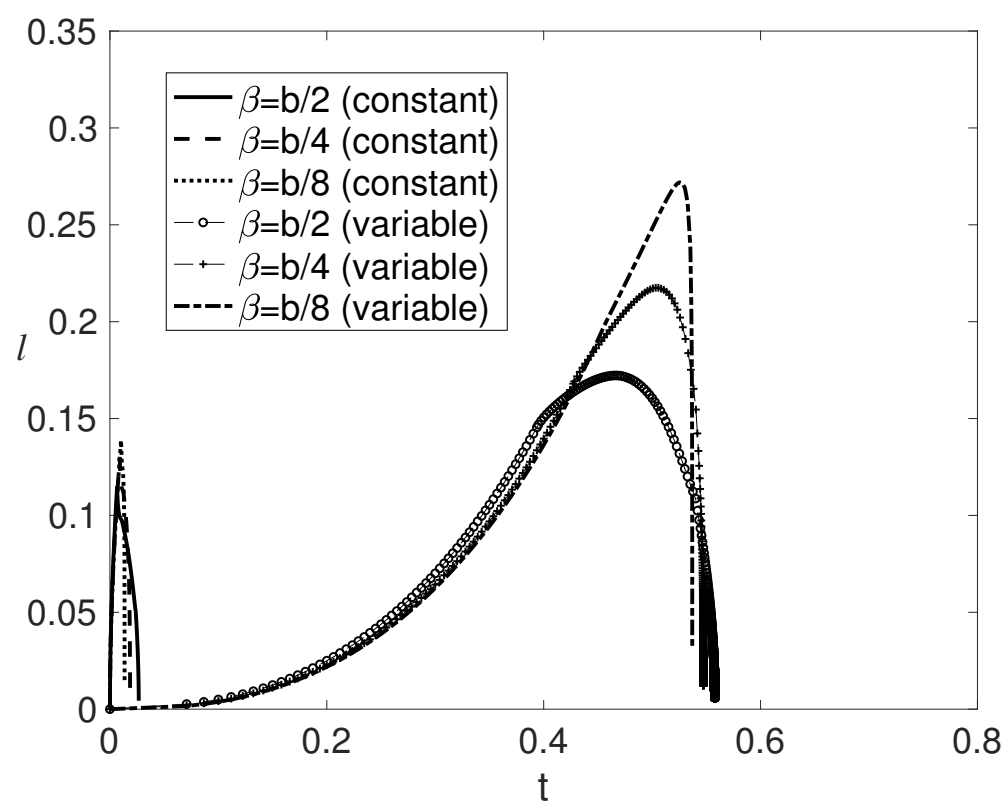

Figure 13: CZ length vs. time for $b=4$ (viscoelastic case). 


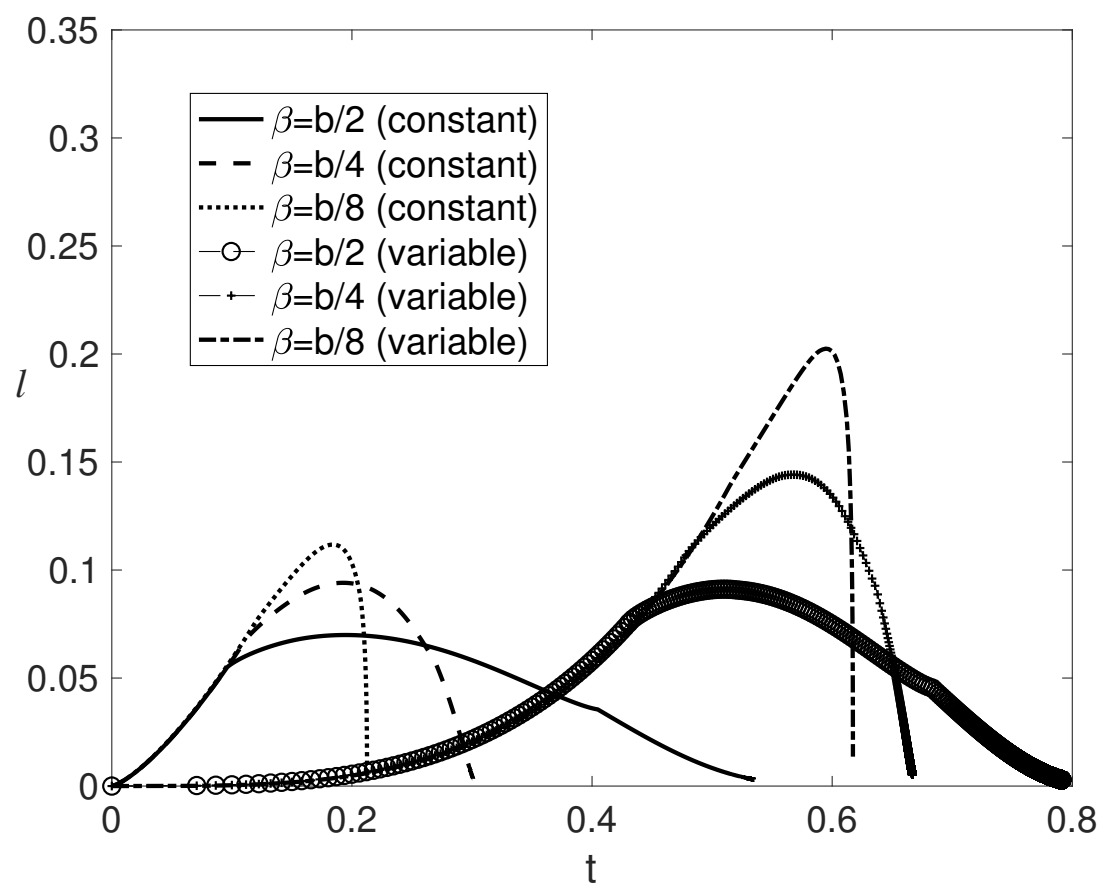

Figure 14: CZ length vs. time for $b=1.5$ (viscoelastic case).

The results for the propagating crack stage show significant differences while comparing the case of a constant load with that of a variable load. In the variable loading case, the crack begins to grow at a much later time than in the constant loading case, as expected.

For the variable loading case, Figs. 15 and 16 illustrate the $\mathrm{CZ}$ and crack evolution for $b=18$, which is of the same order as for PMMA, see ([6], Appendix $\mathrm{A})$, and $\beta=b / 2=9$. It is evident from these figures that, when $b$ is large, the crack grows very rapidly, so that the delay time, $t_{d}$, is rather close to the the rupture time, $t_{r}$, cf. also Fig. 6 . 


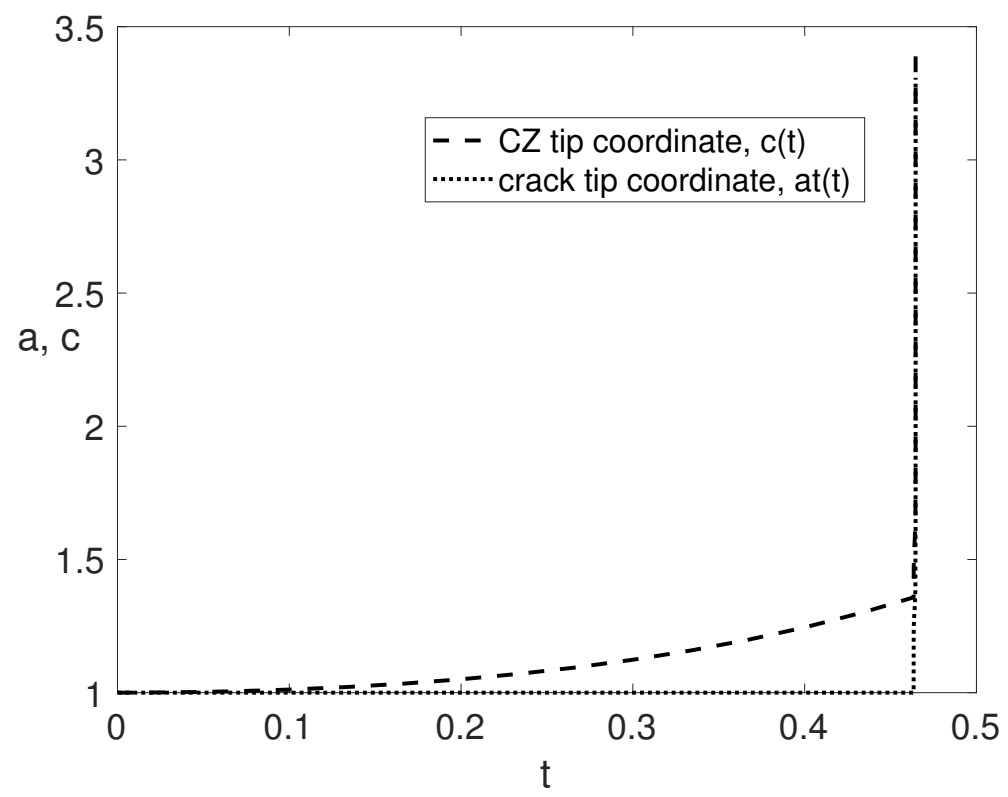

Figure 15: $a(t)$ and $c(t)$ for $b=18, \beta=9$ (variable loading, elastic case).

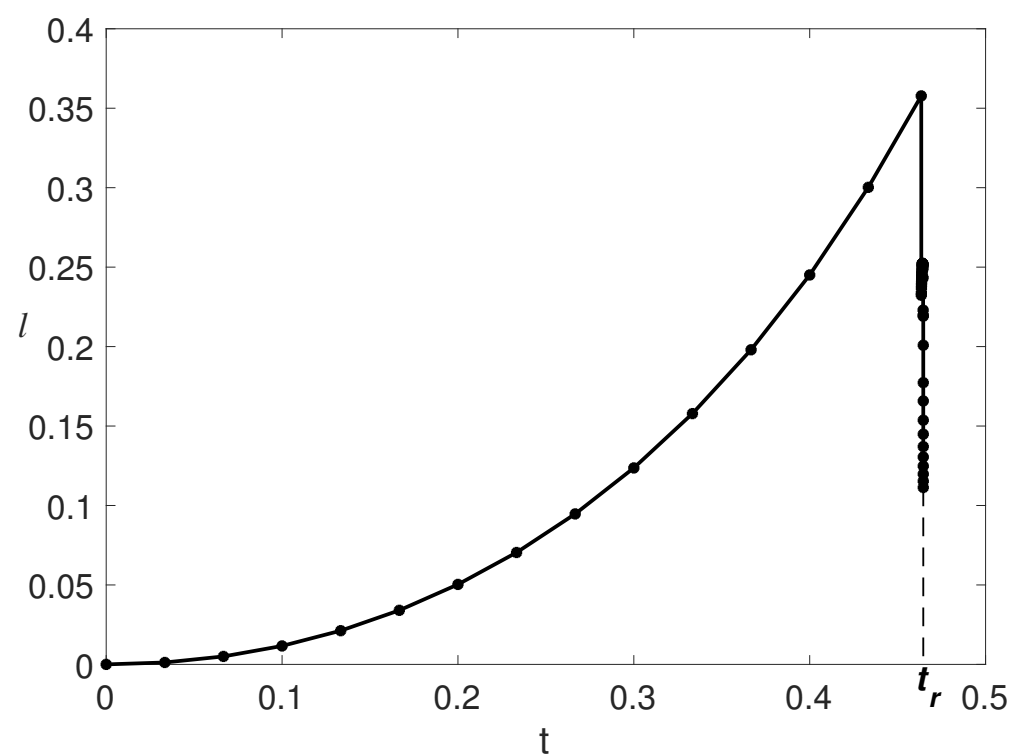

Figure 16: CZ length $l=c(t)-a(t)$ vs. time for $b=18, \beta=9$ (variable loading, elastic case). 
In [6, a detailed inspection of the onset of crack growth was given under constant external load. It has been observed that for some parameter sets, the crack growth starts by an instant crack length jump followed by a continuous growth. Fig. 17, where we present the graph of the crack length at the onset of crack growth in more details, shows that this effect holds also in the variable load case.

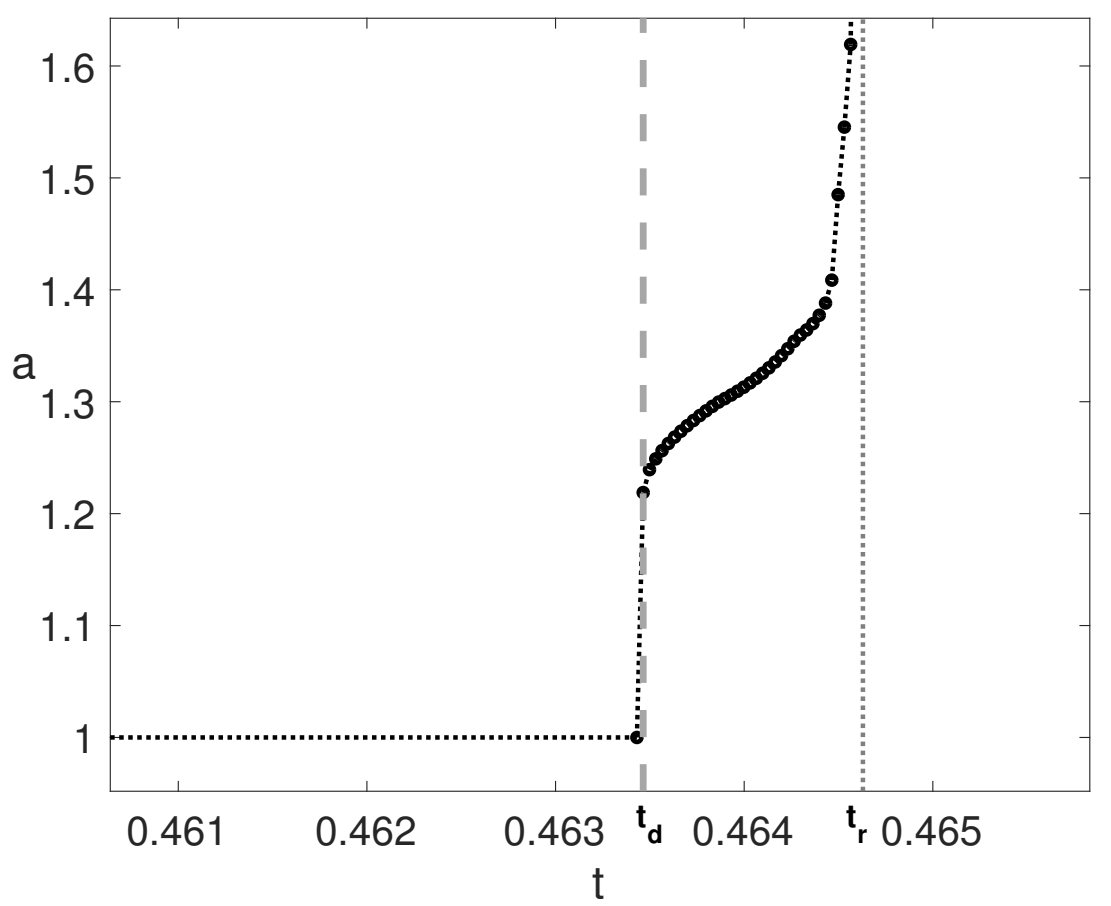

Figure 17: Crack length vs. time, near the delay time, for $b=18, \beta=9$, (variable loading, elastic case).

\section{Conclusions}

We can draw the following conclusions from the obtained results for constant and variable external load.

- A novel non-linear history-dependent generalisation of the Leonov-PanasyukDugdale cohesive zone model of crack propagation in linearly elastic and visco-elastic materials was presented in the paper, extending to the variable external load the results of [6] for the constant external load case. The obtained results show that the CZ model with the history-dependent yield condition (1)-(2) on the cohesive zone can be used for numerical simulation of the crack propagation under both, constant and variable loads. 
- After the crack starts, the crack growth rate increases, while the CZ length decreases with time.

- The time, when the CZ length decreases to zero seems to coincide with the time, when the crack length becomes infinite and can be associated with the complete rupture of the body.

- In the elastic case for some material parameters, there is an unstable crack growth (a jump) at the onset of crack propagation, followed by a stable crack growth.

- Employing a similar approach, the problem described in this article can be generalised in several directions. First, the external loading with a more general time-dependence, e.g., periodic or polynomial in time, can be analysed. Second, the history-dependent CZ model with time-variable loading can be implemented in three-dimensional problems with a penny-shaped crack with a 2D cohesive zone attached to the crack front, geometrically similar to the setting in [15. For bodies and cracks having more general shapes, when there is no analytic solution for the bulk of material, the history-dependent CZM can be still used if some general numerical methods like Boundary Element Method, Finite Element Method, Mesh Free Methods, etc. are implemented, cf. [1, 13. Third, the transient dynamic crack propagation can be also considered, e.g., similar to [9] but without the assumption of infinite cohesive zone.

\section{References}

[1] Allen D.H, Searcy C.R. A micromechanical model for a viscoelastic cohesive zone. Intern. J. Fracture, Vol. 107, pp. 159-176, 2001.

[2] Cotterell B., Fracture and life, World Scientific, Singapore, 2010.

[3] Dugdale D.S., Yielding of steel sheets containing slits, J. Mech. Phys. Solids, Vol. 8, 100-104, 1960.

[4] Hakim L., Numerical Implementation of a Cohesive Zone Model for Time and History Dependent Materials, PhD Thesis, Brunel University London, 2014.

[5] Hakim L, Mikhailov S.E., Nonlinear Abel type integral equation in modelling creep crack propagation, Integral Methods in Science and Engineering: Computational and Analytic Aspects, editors: C. Constanda C. and P. Harris, Springer, 191-201, 2011.

[6] Hakim L., Mikhailov S.E. Integral equations of a cohesive zone model for history-dependent materials and their numerical solution, Quart. J. Mechanics and Applied Math., Vol. 68, 387-419, 2015, doi: 10.1093/qj$\mathrm{mam} / \mathrm{hbv} 013$. 
[7] Hakim L., Mikhailov S.E. Integral Equations in Cohesive Zones Modelling of Fracture in History Dependent Materials, In: Proceedings, World Congress on Engineering 2013, WCE 2013. Newswood Limited, International Association of Engineers, ISBN 978-0-988-19251-0-7, 2013, 226-231

[8] Leonov M.Ya., Panasyuk V.V., Development of the smallest cracks in the solid, Applied Mechanics (Prikladnaya Mekhanika), Vol. 5, No. 4, 391-401, 1959 .

[9] Leise T.L, Walton J.R., Gorb Y. A boundary integral method for a dynamic, transient mode I crack problem with viscoelastic cohesive zone, Int. J. Fract., Vol. 162, 69-76, 2010.

[10] Mikhailov S. E., Namestnikova I. V. History-sensitive accumulation rules for life-time prediction under variable loading, Archive of Applied Mechanics, Vol. 81, 1679-1696, 2011, DOI 10.1007/s00419-011-0511-6.

[11] Mikhailov S.E., Namestnikova I.V., Local and non-local approaches to creep crack initiation and propagation, Proceedings of the 9th International Conference on the Mechanical Behaviour of Materials, Geneva, Switzerland, 2003.

[12] Muskhelishvili N.I., Some Basic Problems of the Mathematical Theory of Elasticity, Noordhoff International Publishing, The Netherlands, 1954.

[13] Rabczuk T., Areias P.M.A., Belytschko T. A simplified meshfree method for shear bands with cohesive surfaces, International Journal for Numerical Methods in Engineering, Vol. 69, 993-1021, 2007.

[14] Rabotnov Yu. N. Elements of hereditary solid mechanics, Mir Publishers, Russia, 1977.

[15] Wnuk M.P., Knauss W.G., Delayed fracture in viscoelastic-plastic solids, Int. J. Solids Structures, Vol. 6, 995-1009, 1970. 